\title{
Response: Authors' Reply to Adding Particulate or Non-Particulate Steroids to the Local Anesthetics When Performing Parasagittal Interlaminar Epidural Injections
}

We thank the Knezevic et al for their great interest in our published article and their compliments and comments(1). We also agree with authors that parasagittal interlaminar (PIL) approach is under presented in the literature and is not frequently differentiated from midline interlaminar epidural injections.

The authors have enquired regarding the differential efficacy of particulate versus non-particulate steroids. It must be mentioned here that both betamethasone and methylprednisolone are particulate steroids though the particle size of betamethasone is bit smaller (2). We agree with the authors of this letter that one of the reasons for the difference in our study results and previous studies conducted by Manchikanti et al could be because of the use of methylprednisolone versus betamethasone.

About the detail of analysis of ventral epidural spread, lateral images were taken to evaluate the ventral epidural space. Ventral spread was defined as present if contrast travelled along the posterior longitudinal ligament or abutted the posterior aspect of the contiguous vertebral body at the level of needle insertion $(3,4)$. The detail was not included in the manuscript because of limited word count. Moreover, the possibility of better "wash-out" of inflammatory mediators because of the use of higher volume of injectate contributing to the efficacy cannot be ruled out in our study. However, no difference has been found between small and large volume of injectate of epidural steroids in lumbosacral pain using transforaminal techniques by earlier studies (5).

\author{
Babita Ghai, MD \\ Additional Professor \\ Department of Anaesthesia and \\ Intensive Care \\ PGIMER, Sector-12 \\ Chandigarh, India-160012 \\ E-mail: ghaibabita1@gmail.com \\ Dipika Bansal, MD \\ Assistant Professor \\ Department of Pharmacy Practice \\ National Institute of Pharmaceutical \\ Education and Research \\ S.A.S. Nagar (Mohali)-160062 \\ Punjab, India \\ E-mail: dipikabansal079@gmail.com \\ Sarvdeep Singh Dhatt, MD \\ Department of Orthopaedics \\ Post Graduate Institute of Medical Education \\ and Research \\ Chandigarh, India
}

\section{Reference}

1. Ghai B, Kumar K, Bansal D, Dhatt SS, Kanukula R, Batra YK. Effectiveness of parasagittal interlaminar epidural lo- 3 . cal anesthetic with or without steroid in chronic lumbosacral pain: A randomized, double-blind clinical trial. Pain Physician 2015; 18:237- 248.

2. Ghai B, Bansal D, Kay JP, Vadaje KS, Wig J. Transforaminal versus parasagittal interlaminar epidural steroid injection in 4 . low back pain with radicular pain: A ran- domized, double-blind, active-control trial. Pain Physician 2014; 17:277-290.

3. Ghai B, Vadaje KS, Wig J, Dhillon MS. Lateral parasagittal versus midline interlaminar lumbar epidural steroid injection for management of low back pain with lumbosacral radicular pain: a double-blind, randomized study. Anesth Analg 2013; 117:219-27.

Park $\mathrm{CH}$, Lee SH, Kim BI Comparison of the effectiveness of lumbar transforaminal epidural injection with particulate and nonparticulate corticosteroids in lumbar radiating pain. Pain Medicine 2010; 11:1654-1658.

5. Lee JH, Moon J, Lee SH Comparison of effectiveness according to different approaches of epidural steroid injection in lumbosacral herniated disk and spinal stenosis. J Back Musculoskelet Rehabil 2009; 22:83-89. 